\title{
ACCURACY ASSESSMENT OF A COMPLEX BUILDING 3D MODEL RECONSTRUCTED FROM IMAGES ACQUIRED WITH A LOW-COST UAS
}

\author{
E. Oniga ${ }^{\mathrm{a}}$, C. Chirilăa ${ }^{\mathrm{a}}$, F. Stătescu ${ }^{\mathrm{b}}$ \\ ${ }^{a}$ Department of Terrestrial Measurements and Cadastre, "Gheorghe Asachi” Technical University of Iasi, D. Mangeron \\ Street, no.65, Iasi, Romania - ersilia.oniga@tuiasi.ro, constantin.chirila@tuiasi.ro \\ ${ }^{b}$ Department of Hydro Amelioration and Environmental Protection, "Gheorghe Asachi" Technical University of Iasi, \\ D. Mangeron Street, no. 65, Iasi, Romania - fstatesc@yahoo.fr
}

\section{Commission II}

KEY WORDS: Building 3D Model, hyperbolic paraboloid, low-cost UASs, TLS, accuracy assessment

\begin{abstract}
:
Nowadays, Unmanned Aerial Systems (UASs) are a wide used technique for acquisition in order to create buildings 3D models, providing the acquisition of a high number of images at very high resolution or video sequences, in a very short time. Since low-cost UASs are preferred, the accuracy of a building 3D model created using this platforms must be evaluated. To achieve results, the dean's office building from the Faculty of "Hydrotechnical Engineering, Geodesy and Environmental Engineering" of Iasi, Romania, has been chosen, which is a complex shape building with the roof formed of two hyperbolic paraboloids. Seven points were placed on the ground around the building, three of them being used as GCPs, while the remaining four as Check points (CPs) for accuracy assessment. Additionally, the coordinates of 10 natural CPs representing the building characteristic points were measured with a Leica TCR 405 total station. The building 3D model was created as a point cloud which was automatically generated based on digital images acquired with the low-cost UASs, using the image matching algorithm and different software like 3DF Zephyr, Visual SfM, PhotoModeler Scanner and Drone2Map for ArcGIS. Except for the PhotoModeler Scanner software, the interior and exterior orientation parameters were determined simultaneously by solving a self-calibrating bundle adjustment. Based on the UAS point clouds, automatically generated by using the above mentioned software and GNSS data respectively, the parameters of the east side hyperbolic paraboloid were calculated using the least squares method and a statistical blunder detection. Then, in order to assess the accuracy of the building 3D model, several comparisons were made for the facades and the roof with reference data, considered with minimum errors: TLS mesh for the facades and GNSS mesh for the roof. Finally, the front facade of the building was created in 3D based on its characteristic points using the PhotoModeler Scanner software, resulting a CAD (Computer Aided Design) model. The results showed the high potential of using low-cost UASs for building 3D model creation and if the building 3D model is created based on its characteristic points the accuracy is significantly improved.
\end{abstract}

\section{INTRODUCTION}

Nowadays, Unmanned Aerial Systems (UASs) are a widely used technique for acquisition in order to create building 3D models, providing the acquisition of a high number of images at very high resolutions or video sequences. Using mathematical formulation implemented into a big number of software, commercial and open-source, the 2D coordinates of common features identified by image matching algorithm are transformed into 3D coordinates. Images contain all the useful information to form geometry and texture for a 3D modelling application. But the reconstruction of detailed, accurate and photo-realistic 3D models from images is still a difficult task, particularly in the case of large and complex sites that have to be photographed with widely separated or convergent image blocks (Barazetti et al., 2010).

Since low-cost UASs are preferred, being affordable to any user, and different packages are available on the market, as open-source, free web service or as commercial software, the accuracy of a building 3D model created using this platforms and different software must be evaluated.

Over the years many studies have been conducted regarding the accuracy of image-based $3 \mathrm{D}$ models by comparing them with reference data, usually the TLS point cloud. The 3D models, represented as point cloud or mesh surfaces, have been automatically generated from digital images using different software.

Point clouds and surface models automatically generated from terrestrial digital imagery using the open-source software Bundler/PMVS2, free software VisualSFM, Autodesk Web Service 123D Catch beta and low-cost software Agisoft PhotoScan were compared with the results from terrestrial laser scanning, concluding that the models derived from imagery could not achieve the same quality of geometrical accuracy as laser scanning (Kersten et al., 2015).

UAS images taken with camera's optical axis in vertical and oblique position or architectural cultural heritage reconstruction, were processed by the commercial software PhotoScan of Agisoft the results being good in terms of accuracy (average error $<2 \mathrm{~cm}$ ) and reliability (Bolognesi et al., 2015).

In Remondino et al. (2012) an investigation of automated image orientation packages, i.e Agisoft, Photosynth, Bundler, Apero and Visual SfM is presented, in order to clarify potentialities and performances when dealing with large and complex datasets. 


\section{STUDY AREA AND DATA AQUISITION}

For this case study the dean's office building from the Faculty of "Hydrotechnical Engineering, Geodesy and Environmental Engineering"- "Gheorghe Asachi" Technical University of Iasi, Romania has been chosen, i.e. a complex shape building with a roof structure having a shape of a hyperbolic paraboloid.

In order to assess the accuracy of the building under study $3 \mathrm{D}$ model, several comparisons will be made for the facades and the roof with reference data, considered with minimum errors. So, a TLS point cloud (Figure 1) will be considered as reference data for the facades. This was the result of registering five point clouds acquired with a Leica ScanStation 2 terrestrial laser scanner, using the direct georeferencing process (Oniga et al., 2012).

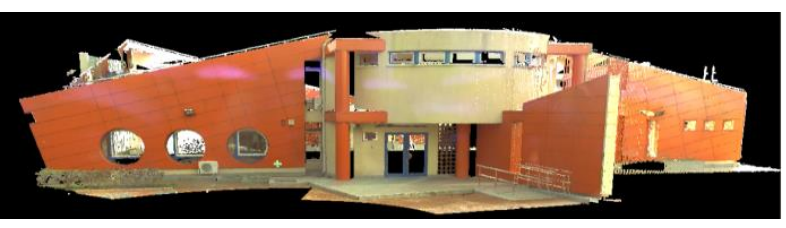

Figure 1. The TLS point cloud with realistic colour information

So, in order to obtain accurate results in the comparisons steps, the TLS point cloud was filtered, i.e. the points that do not belong to the building of interest were eliminated, namely: air conditioning, lighting sources or external stairs mounted on the facades of the building, stormwater drain tubes and also points measured inside the building through its windows. Based on the remaining points, a mesh surface was created using the "I-Site Studio" software and the "Spherical triangulation" function (Figure 2).

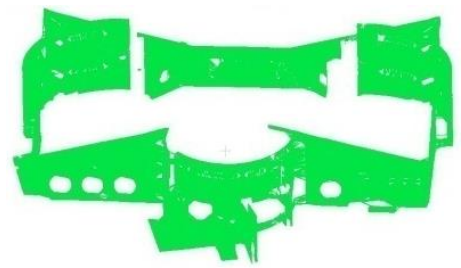

(a)

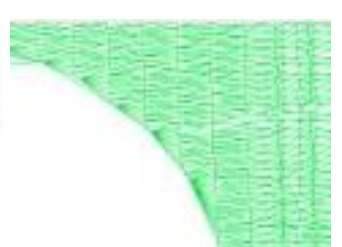

(b)
Figure 2. The TLS mesh created after the filtering process (a), detail (b)

Taking into account that the roof could not be scanned from the ground, 903 points were measured on its surface and limits using the GNSS technology being further considered as reference data for the roof.

The low-cost UAS platform used in this paper was a DJI Phantom 3 Standard which has a built-in camera with 3-axis stabilization gimbal that captures sharp, vivid $2.7 \mathrm{~K}$ Ultra HD video at 30 frames per second and a maximum of $40 \mathrm{Mbps}$. The digital camera is equipped with a $6.2031 \mathrm{~mm}$ by $4.6515 \mathrm{~mm}$ image sensor capable of taking images with a resolution of 12 MP and 4000 x 3000 pixels (Oniga et al., 2016).

In order to acquire the UAS images for the building 3D model creation, the flight was done in manual mode in approximately 16 minutes. In order to assure a regular and higher overlap in the image block, the building was photographed all around at an altitude of approximately $15 \mathrm{~m}$ above the ground, 63 images being taken from 63 different camera positions distributed circularly around the building.
To obtain accurate information when using a low-cost UASs, the indirect georeferencing process must be used, the placing and measuring of Ground Control points (GCPs) being mandatory. For this case study, the GCPs were made by plexiglass, having the centre marked by the intersection of two black triangles and a metal bold. Seven points were placed on the ground around the building, three of them being used as GCPs, while the remaining four as Check points (CPs) for accuracy assessment. Then, their coordinates were measured with high accuracy using the GNSS technology. Additionally, the coordinates of 10 natural CPs representing the building characteristic points i.e corners of windows and doors, were measured with a Leica TCR 405 total station.

\section{CREATING THE 3D MODEL OF THE BUILDING}

3.1 The 3D model generation of the "Dean's office" building based on UAS images, using the Zephyr software

3.1.1 Photogrammetric data processing: The building 3D reconstruction process includes a series of steps presented in Oniga et al. (2016).

For this case study, a free-network approach in the bundle adjustment was applied, the result being the three-dimensional coordinates of common features identified by image matching algorithm, in a local coordinate system. The interior and exterior orientation parameters for each camera position were determined simultaneously by solving a self-calibrating bundle adjustment (Nex \& Remondino, 2013). So, in a first step, a point cloud was automatically generated containing a number of 285542 points (Figure 3a) and in a second step, a textured mesh surface was automatically created based on the point cloud containing 6085681 triangles in approximately $8 \mathrm{~h}$ (Figure $3 \mathrm{~b}$ ).

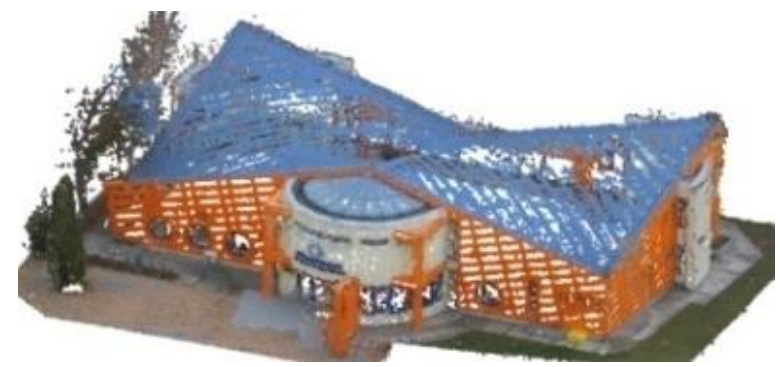

(a)

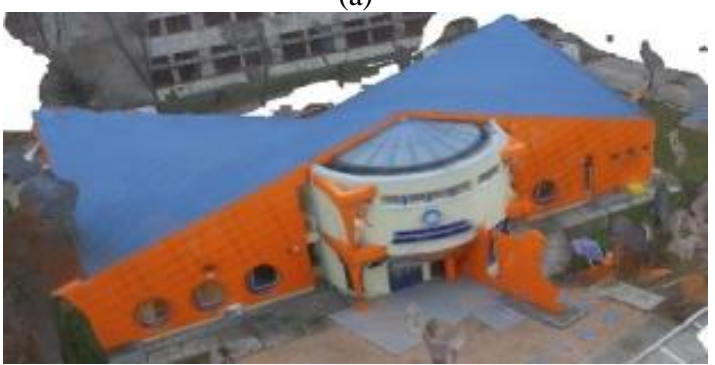

(b)

Figure 3. The „Dean's office” building model, created in the Zefhyr software based on UAS images point cloud (a), mesh surface (b)

The options "Very high" for the keypoints density, "Full" for bundle adjustment and "Sequential" for the photos ordering were chosen.

Analysing the point cloud we can see that there is some missing data on the roof surface and the facades. 
Only at the end of the bundle adjustment a similarity (Helmert) transformation was performed in order to bring the image network results into the desired reference coordinate system, i.e national "Stereographic - 1970". As previously demonstrated in Oniga et al. (2016), if no constraint is introduced in the process of bundle adjustment, increasing the GCPs number will not improve the $3 \mathrm{D}$ shape of the surveyed scene. So, in this situation, the minimum number of three artificial GCPs mentioned above was used, their coordinates being manually measured on the oriented images. The final mean residual was $0.021 \mathrm{~m}$.

The computer used for this data processing was: PC Asus, Intel Core i5-3230M CPU @ 2.60GHz, 4 GB RAM, GPU: Intel(R) HD Graphics 4000, NVIDIA GeForce GT 720M.

3.1.2 Quality assessment: In order to assess the accuracy of the building 3D model, first the coordinates of the CPs were measured on oriented images and compared with the ones determined with high accuracy using the GNSS technology.

In Figure 4 we can see the position of the GCP 1, 2 and 3, the four Artificial CPs 1, 2, 3 and 4 together with camera positions and orientations.

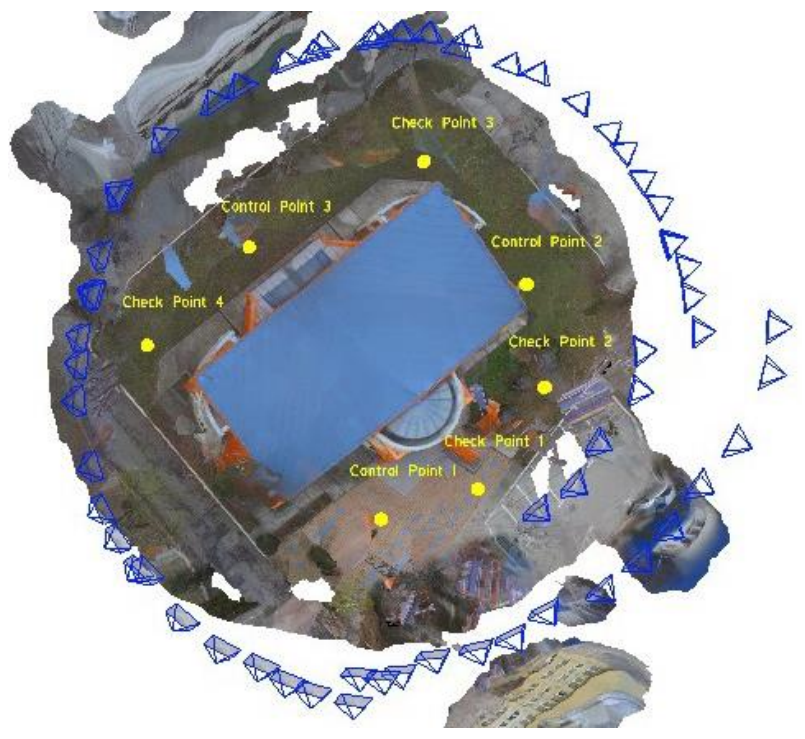

Figure 4. The visualization of the Control Points 1, 2 and 3, the Artificial Check points 1, 2, 3 and 4 and the camera positions and orientations

Based on the differences between the $\mathrm{CP}$ coordinates, the residual was calculated, which is the Euclidian distance between the two coordinate sets for a point calculated with the distance equation:

$$
D=\sqrt{\left(X_{r}-X_{i}\right)^{2}+\left(Y_{r}-Y_{i}\right)^{2}+\left(Z_{r}-Z_{i}\right)^{2}}
$$

where:

$\left(\mathbf{X}_{\mathbf{r}}, \mathbf{Y}_{\mathbf{r}}, \mathbf{Z}_{\mathbf{r}}\right)$ - the coordinates of a CP computed after the GNSS measurements;

$\left(\mathbf{X}_{\mathbf{i}}, \mathbf{Y}_{\mathbf{i}}, \mathbf{Z}_{\mathbf{i}}\right)$ - the coordinates of a CP computed based on image measurements.

The residuals (D) of the artificial CPs after georeferencing the point cloud are listed in Table 1.

\begin{tabular}{|c|c|c|c|c|}
\hline \multirow{2}{*}{ Check point } & \multicolumn{3}{|c|}{ Differences } & Distance \\
\cline { 2 - 5 } & $\mathbf{\Delta X}[\mathbf{m}]$ & $\boldsymbol{\Delta} \mathbf{Y}[\mathbf{m}]$ & $\boldsymbol{\Delta Z}[\mathbf{m}]$ & $\mathbf{D}[\mathbf{m}]$ \\
\hline 1 & 0.005 & 0.011 & -0.003 & 0.012 \\
\hline 2 & 0.011 & 0.006 & -0.017 & 0.021 \\
\hline 3 & -0.006 & -0.012 & 0.018 & 0.022 \\
\hline 4 & 0.030 & -0.006 & 0.003 & 0.031 \\
\hline RMS & $\mathbf{0 . 0 1 6}$ & $\mathbf{0 . 0 0 9}$ & $\mathbf{0 . 0 1 3}$ & $\mathbf{0 . 0 2 3}$ \\
\hline
\end{tabular}

Table 1: The residuals of the artificial CPs after georeferencing the point cloud using the minimum number of control points

The RMS (Root Mean Square) values along the axis and cumulative were calculated: RMS along the $\mathrm{X}$ axis $(0.016 \mathrm{~m})$, RMS along the $\mathrm{Y}$ axis $(0.009 \mathrm{~m})$, RMS along the $\mathrm{Z}$ axis $(0.013$ $\mathrm{m})$, spatial RMS $(0.023 \mathrm{~m})$.

3.2 The 3D model generation of the „Dean's office" building based on UAS images, using the Visual SfM software

3.2.1 Photogrammetric data processing: The first step when processing the images into Visual SfM is to add the images into the SfM Workspace. Then, the feature detection and full pairwise image matching functions were run and, at the end, the sparse reconstruction and dense reconstruction by using Yasutaka Furukawa's CMVS were performed.

Since the images resolution is $4000 \times 3000$ pixels, SiftGPU actually starts the detection with size 2000 . So, the parameter was changed by using "Tools -> Enable GPU -> Set Maximum DIM" function. The result was obtained in 17 minutes on a high performance computer (Intel Core i7-3820 CPU @ 3.60GHz, 64 GB RAM, NVIDIA Quadro K1200, using the operating system Windows 10, 64 bits).

The computer used for processing was different from the computer used for the other software packages processing, because the image data processing using the laptop was cancelled after 3 days.

The result was a dense point cloud containing 958,966 points, represented in a local coordinate system (Figure 5).

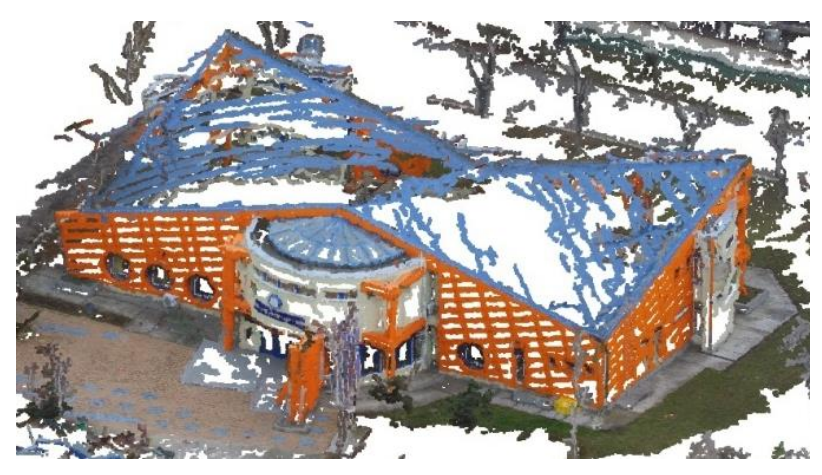

Figure 5. The „Dean's office” building model created in the Visual SfM software based on UAS images, represented as a point cloud

In order to bring the point cloud into the desired reference coordinate system, i.e. "Stereographic - 1970", three control points were used, i.e. Control Point 1, 2 and Check Point 1. We chose the Check Point 1 instead of GCP 3 , because no points were generated in the area where this control point was placed, due to trees. The process was conducted into CloudCompare software using the function "Align (point pairs picking)" from 
the "Registration" menu. The coordinates of the control points were measured directly on the point cloud being obtained a RMS of $0.021 \mathrm{~m}$ for the alignment process.

Visually analysing the point cloud we can see that on the back side of the building and on the right side, the point cloud is very disturbed. The main cause is the existence of the trees, which, even if not leafy trees, have obscured the building facades. We can also see that there are missing points on the roof surface, on the facades and on the white parts of the building, all these having uniform textures.

3.2.2 Quality assessment: In this case the coordinates of the remaining three check points couldn't be measured because no points were generated in their areas due to trees.

\subsection{The 3D model generation based on UAS images, using the PhotoModeler Scanner software}

3.3.1 Photogrammetric data acquisition and processing: For the data processing with "PhotoModeler Scanner 2013" software the image data set was reduced to 48 UAS images which were imported into the software. For this case study the "Automated project" was selected and the option of feature points detection and referencing was chosen.

TEST 1: The project was processed without specifying the type of camera and the calibration parameters, so for the camera parameters an approximation was made based on the EXIF information.

The option "High" for the points density and "Ordered photos Surround" for the photos ordering were chosen.

Following the bundle adjustment process, the PhotoModeler Scanner software calculated the three-dimensional coordinates of 6112 points (sparse point cloud) in a local coordinate system as well as the exterior orientation parameters for each camera position.

To define the world coordinate system the coordinates of three artificial control points were introduced, previously used into Zephyr software. When manually marking the points on images, a large residual was obtained, concluding that the images haven't been correctly oriented based only on EXIF information. So, we chose not to make any further steps.

TEST 2: PhotoModeler normally requires information on the camera (focal length, main point and format size) before the images processing. Having analysed the process done in TEST 1 , the project was processed specifying the type of camera and the calibration parameters. The camera was previously calibrated using a 2D calibration grid, i.e. the A4 sheet found in the PhotoModeler Scanner library.

Following the bundle adjustment process, the "PhotoModeler Scanner" software calculated the three-dimensional coordinates of 13344 points (sparse point cloud) in a local coordinate system as well as the exterior orientation parameters for each camera position.

To define the world coordinate system the same three artificial control points were introduced, a large residual being obtained for the Control Point 3 situated in back side of the building. So, in order to correct the images orientations, 29 points have been manually referenced and the project was processed. The overall residual of the project was 0.704 pixels, less than the recommended 5 pixels and the maximum residual of points marking was 3.76 pixels. The cumulative RMS of CPs was $0.017 \mathrm{~m}$.

Finally, the creation of a dense points cloud option was chosen (dense reconstruction), the result being a cloud containing 278685 points. Thus, in Figure 6, can be seen the results of the bundle adjustment process, the camera positions and orientations towards the building to be photographed and the textured dense point cloud in the world coordinate system. In Figure 9, can be seen the mesh surface.

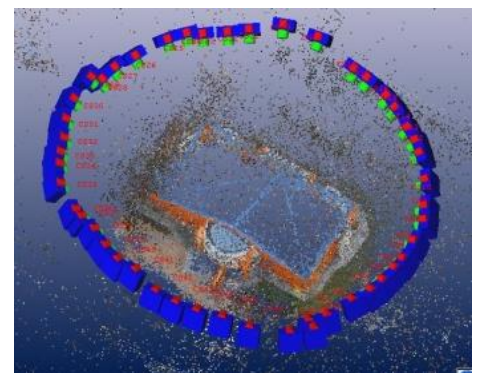

Figure 6. The camera positions and orientations and the dense point cloud

3.3.2 Quality assessment: In order to assess the accuracy of the building 3D model, the coordinates of the CPs were measured on oriented images and compared with the ones determined with high accuracy using the GNSS technology.

The residuals of the artificial CPs after georeferencing the point cloud are listed in Table 2.

\begin{tabular}{|c|c|c|c|c|}
\hline \multirow{2}{*}{ Check point } & \multicolumn{3}{|c|}{ Differences } & Distance \\
\cline { 2 - 5 } & $\boldsymbol{\Delta X}[\mathbf{m}]$ & $\Delta \mathbf{Y}[\mathbf{m}]$ & $\boldsymbol{\Delta Z}[\mathbf{m}]$ & $\mathbf{D}]$ \\
\hline 1 & 0.009 & -0.004 & -0.002 & 0.010 \\
\hline 2 & 0.013 & -0.002 & 0.001 & 0.013 \\
\hline 3 & 0.014 & -0.024 & 0.008 & 0.029 \\
\hline 4 & -0.019 & -0.023 & -0.016 & 0.034 \\
\hline RMS & $\mathbf{0 . 0 1 4}$ & $\mathbf{0 . 0 1 7}$ & $\mathbf{0 . 0 0 9}$ & $\mathbf{0 . 0 2 4}$ \\
\hline
\end{tabular}

Table 2. The residuals of the artificial CPs after georeferencing the point cloud using the minimum number of control points

TEST 3: The front facade of the building was created based on the characteristic building points, using the software specific functions (Figure 7). A number of 189 points were measured on the oriented images using the project created in Test 2.

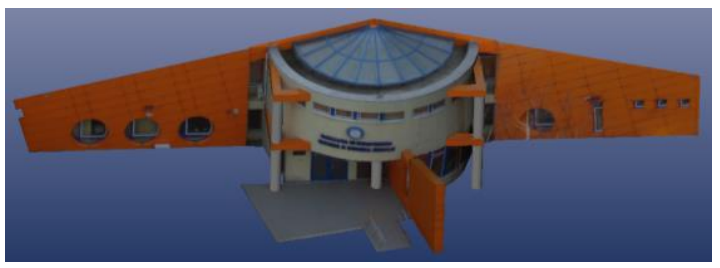

Figure 7. The „Dean's office” front facade modelled in 3D using the PhotoModeler Scanner software functions

3.3.2 Quality assessment: For this case study, all the image coordinate errors were less than 5 pixels tolerance suggested by PhotoModeler Scanner. The overall residual of the project was 3.23 pixels, less than the recommended 5 pixels. The total error for determining the world coordinates, ranges between $4.4 \mathrm{~mm}$ and $5.4 \mathrm{~cm}$. The angles between the projection rays corresponding to each point range between $4^{0} .4512 \div 89^{0} .9923$. 
3.4 The 3D model generation of the "Dean's office" building based on UAS images, using the Drone2Map for ArcGis software

3.4.1 Photogrammetric data processing: The first step when processing the images into Drone2Map is to select a template, in this case "3D Mapping". Then, after creating a new project and defining the coordinate system, the images have been added into the Drone2Map and the processing options have been selected. For the process of initial processing the "full" option for the keypoints image scale and "free flight or terrestrial" for matching image pairs were chosen and the option "use geometry for verified matching" was selected. In the case of point cloud and mesh, the image scale was set to "original", point cloud density to "optimal" and point cloud densification to " $9 \times 9$ pixels".

The process was completed in approximately $5 \mathrm{~h}$ using the PC Asus, being obtained a point cloud containing 17622723 points with an average density (points $/ \mathrm{m}^{3}$ ) of 409.8 (Figure 8 a) and a simplified mesh surface with a number of 763785 faces (Figure $8 \mathrm{~b})$.

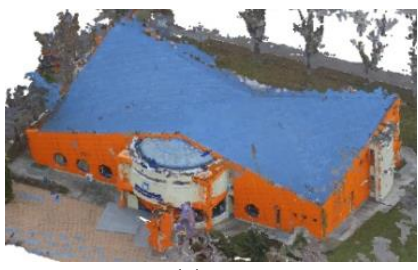

(a)

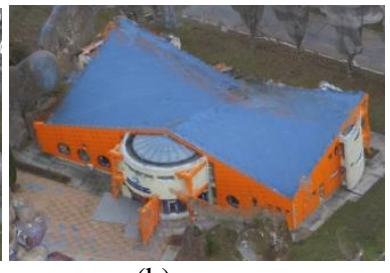

(b)
Figure 8. The „Dean's office” building model, created in the Drone2Map software based on UAS images point cloud (a), mesh surface (b)

3.4.2 Quality assessment: After the process has been completed, the Drone2Map software created a processing report, containing the following main information (Table 3):

\begin{tabular}{|c|l|}
\hline $\begin{array}{c}\text { Average Ground } \\
\text { Sampling Distance }\end{array}$ & $2.36 \mathrm{~cm} / 0.92$ in \\
\hline Images & median of 35690 keypoints per image \\
\hline Camera Optimization & $\begin{array}{l}18.09 \% \text { relative difference between } \\
\text { initial and optimized internal camera } \\
\text { parameters }\end{array}$ \\
\hline Matching & $\begin{array}{l}\text { median of 6816.09 matches per } \\
\text { calibrated image }\end{array}$ \\
\hline Georeferencing & $\begin{array}{l}\text { yes, 3 GCPs (3 3D), } \\
\text { mean RMS error =0.004 m }\end{array}$ \\
\hline $\begin{array}{c}\text { Mean Reprojection } \\
\text { Error [pixels] }\end{array}$ & 0.243686 \\
\hline
\end{tabular}

Table 3. Quality check of the image processing using the Drone2Map software

The residuals of the artificial CPs after georeferencing the point cloud are listed in Table 4.

\begin{tabular}{|c|c|c|c|c|}
\hline \multirow{2}{*}{ Check point } & \multicolumn{3}{|c|}{ Differences } & \multirow{2}{*}{$\begin{array}{c}\text { Distance } \\
\text { D }[\mathrm{m}]\end{array}$} \\
\hline & $\Delta \mathbf{X}[\mathbf{m}]$ & $\Delta \mathbf{Y}[\mathbf{m}]$ & $\Delta \mathbf{Z}[\mathrm{m}]$ & \\
\hline 1 & 0.014 & -0.015 & -0.014 & 0.025 \\
\hline 2 & 0.000 & -0.010 & 0.005 & 0.011 \\
\hline 3 & -0.018 & 0.015 & -0.011 & 0.026 \\
\hline 4 & -0.027 & -0.006 & -0.011 & 0.030 \\
\hline RMS & 0.018 & 0.012 & 0.011 & 0.024 \\
\hline
\end{tabular}

Table 4. The residuals of the artificial CPs after georeferencing the point cloud using the minimum number of control points

\subsection{Modelling the building roof}

In order to approximate the roof's structure with the most probable geometric form of a hyperbolic paraboloid, the computation steps can be found in Oniga et al. (2012).

The hyperbolic paraboloid parameters were calculated based on GNSS data and the points clouds automatically generated by different software, namely: Zephyr, Visual SfM and PhotoModeler Scanner. An application in MATLAB programming language was made for the calculation.

To calculate the parameters of the East hyperbolic paraboloid, a total of 396 GNSS points were used. After going through three iterations, the maximum correction calculated for the normal altitudes along the $\mathrm{Z}$ axis of the hyperbolic paraboloid was $-\mathbf{0 . 1 6 7 4 ~ m . ~}$

The parameters $\boldsymbol{a}$ and $\boldsymbol{b}$ of the hyperbolic paraboloid resulting from the adjustment process by means of indirect observations can be affected by gross errors. The corrections resulting from the adjustment process should not be used directly to identify large errors. The residuals result not only due to errors in the observations but also because of the functional model error compensation.

\section{Statistical blunder detection applied to the GNSS data}

The concept of statistical blunder detection in surveying was introduced in the mid-1960s and utilizes the cofactor matrix $\mathrm{Q}_{\mathrm{vv}}$ of the residuals (Ghilani and Wolf, 2006).

The equation for blunders rejection is:

$$
\overline{v_{i}}=\frac{\left|v_{i}\right|}{\sqrt{q_{i i}}}>s_{o} \cdot \text { rejection level }
$$

where $\overline{v_{i}}$ are the standardized residuals, $v_{i}$ are the computed residuals and $\mathrm{q}_{\mathrm{ii}}$ the diagonal element of the $\mathrm{Q}_{\mathrm{vv}}$ matrix.

For this case study the rejection level from the Equation (2) was considered 2.8, the value of the confidence level of $95 \%$, the GNSS points whose standardized residual values exceed 0.1482 , being eliminated. After applying this threshold, three GNSS points have been eliminated.

The adjusted parameters of the hyperbolic paraboloid that best fit the 393 GNSS points, remaining after the statistical test application, are:

$$
a=5.1729 \mathrm{~m} ; b=5.6066 \mathrm{~m}
$$

The same calculations were conducted for the automatically generated point clouds, except the Drone2Map point cloud due to the huge number of points, i.e 842088 , the summary being presented in Table 5.

\begin{tabular}{|c|c|c|c|c|}
\hline $\begin{array}{c}\text { Type of } \\
\text { points }\end{array}$ & $\begin{array}{c}\text { Standard } \\
\text { deviation } \\
\text { so }\end{array}$ & $\begin{array}{c}\text { Rejection } \\
\text { value }\end{array}$ & $\begin{array}{c}\text { Number } \\
\text { of initial } \\
\text { points }\end{array}$ & $\begin{array}{c}\text { Number of } \\
\text { eliminated } \\
\text { points }\end{array}$ \\
\hline GNSS & 0.0529 & 0.1482 & 396 & 3 \\
\hline Zephyr & 0.0982 & 0.2749 & 9424 & 71 \\
\hline PhotoModeler & 0.1552 & 0.4345 & 1312 & 6 \\
\hline Visual SfM & 0.2944 & 0.8243 & 10882 & 268 \\
\hline
\end{tabular}

Table 5. The summary of the hyperbolic paraboloid fitting using the least squares principle 


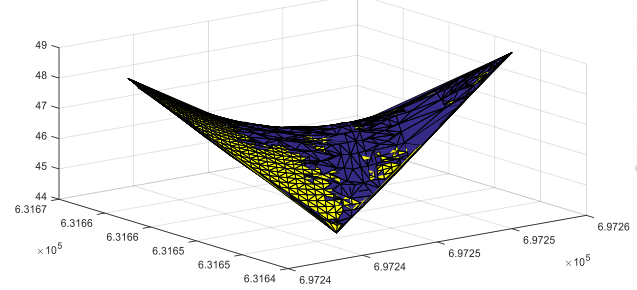

(a)

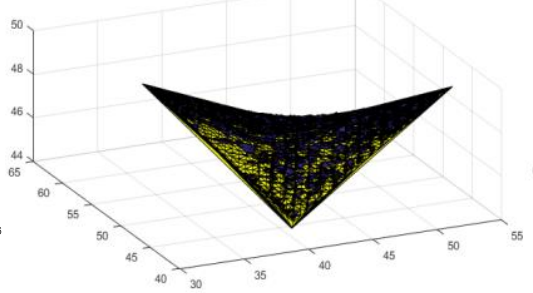

(b)

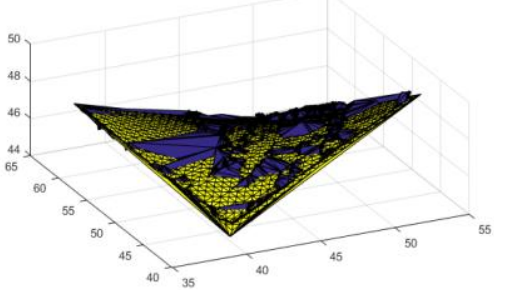

(c)

Figure 9. The roof surface modelled in 3D (blue colour) using the GNSS points (a), Zephyr point cloud (b), Visual SfM point cloud (c) and the best fitting hyperbolic paraboloid shape (yellow colour) from the east side

The parameters $a$ and $b$ of the hyperbolic paraboloid resulting from the adjustment process of the automatically generated point cloud using different software, are listed in Table 6.

\begin{tabular}{|c|c|c|c|}
\hline \multirow{2}{*}{$\begin{array}{c}\text { Type of } \\
\text { points }\end{array}$} & $\begin{array}{c}\text { Maximum } \\
\text { correction for the } \\
\text { normal altitudes } \\
\text { Vz [m] }\end{array}$ & \multicolumn{2}{|c|}{$\begin{array}{c}\text { Parameters } \\
\text { of the east paraboloid }\end{array}$} \\
\cline { 3 - 4 } & -0.0840 & $\mathbf{a}[\mathbf{m}]$ & $\mathbf{b}[\mathbf{m}]$ \\
\hline GNSS & -0.2355 & 5.2596 & 5.6066 \\
\hline Zephyr & -0.2391 & 5.1216 & 5.6461 \\
\hline $\begin{array}{c}\text { PhotoModeler } \\
\text { Scanner }\end{array}$ & 0.8734 & 5.2844 & 5.2367 \\
\hline Visual SfM & & \multicolumn{2}{|c}{} \\
\hline
\end{tabular}

Table 6. The parameters $a$ and $b$ of the hyperbolic paraboloid created based on the roof point clouds

\section{COMPARING THE 3D MODELS OF THE BUILDING}

4.1 Comparing the 3D buildings models based on the characteristic points coordinates

The accuracy of the 3D modelling process was evaluated first by pointing out and verifying the differences between the values of two sets of rectangular coordinates $(X, Y, H)$. These points represent 10 characteristic points of the building (window edges, door, etc.) located on the building facades (Table 7).

The two sets of coordinates, resulted by total station measurements (the reference coordinates) and 3D models interrogation respectively, were created based on UAS images processing using the Zephyr, PhotoModeler Scanner, Drone2Map and Visual SfM software.

To determine the cumulative root mean square value (T) (Eq. 4 ), the following calculations were made: the RMS along the $X$ axis (Rx), the RMS along the $\mathrm{Y}$ axis (Ry) and the RMS along the $\mathrm{Z}$ axis $(\mathrm{Rz})$.

$$
T=\sqrt{R_{x}^{2}+R_{y}^{2}+R_{z}^{2}}
$$

\begin{tabular}{|c|c|c|c|c|}
\hline Soft & $\begin{array}{c}\mathbf{R}_{\mathbf{X}} \\
{[\mathbf{m}]}\end{array}$ & $\begin{array}{c}\mathbf{R}_{\mathbf{Y}} \\
{[\mathbf{m}]}\end{array}$ & $\begin{array}{c}\mathbf{R Z}_{\mathbf{Z}} \\
{[\mathbf{m}]}\end{array}$ & $\begin{array}{c}\mathbf{T} \\
{[\mathbf{m}]}\end{array}$ \\
\hline Zephyr & 0.019 & 0.026 & 0.030 & 0.044 \\
\hline $\begin{array}{c}\text { PhotoModeler } \\
\text { Scanner }\end{array}$ & 0.018 & 0.019 & 0.033 & 0.040 \\
\hline Drones2Map & 0.032 & 0.033 & 0.027 & 0.053 \\
\hline Visual SfM & 0.059 & 0.117 & 0.039 & 0.137 \\
\hline
\end{tabular}

Table 7. The residuals of the 3D models characteristic points coordinates

The $\mathbf{T}$ calculated for the 10 natural check points using the Zephyr and PhotoModeler Scanner software, was evaluated using values of around $\mathbf{4} \mathbf{~ c m}$, confirming that the two survey methods (UAV and total station) are of similar precision, allowing the usage of either of them by convenience, to get similar results. In the case of the Drone2Map and Visual SfM software the residuals are of higher values because the CPs coordinates were measured directly in the point cloud.

\subsection{Comparing the building roof created by different} software

To determine the accuracy of the $3 \mathrm{D}$ point clouds derived by using different software for the building roof, we will use the comparison method between the point cloud and a reference mesh surface, considered for this case study the mesh created based on the GNSS data (Figure 10).

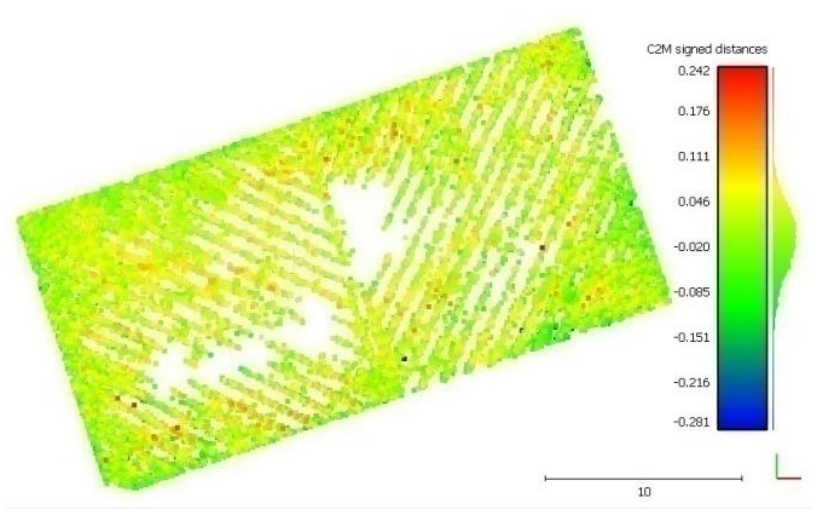

(a)

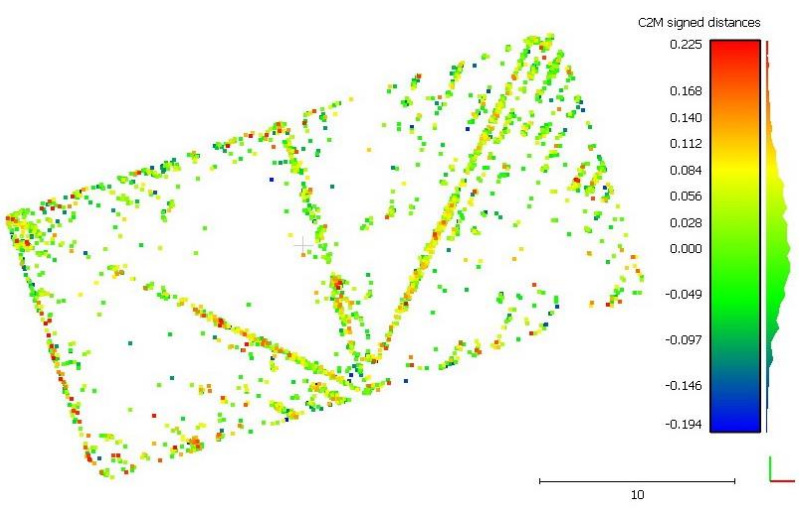

(b) 


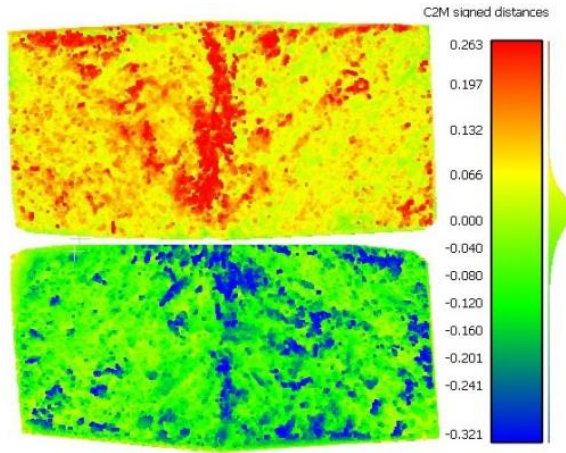

(c)

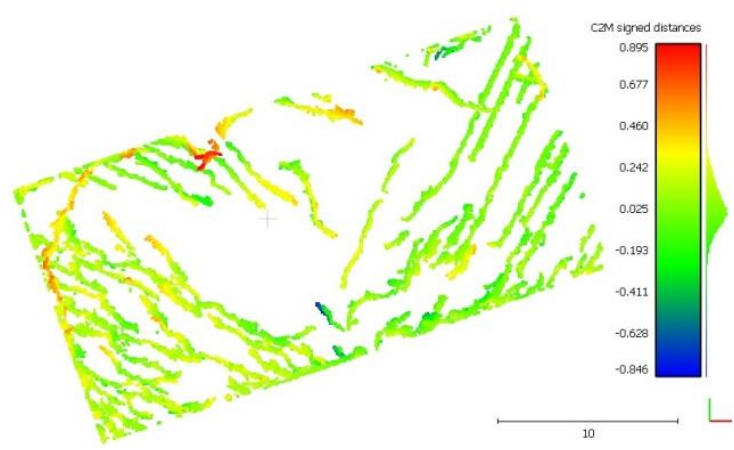

(d)

Figure 10. The Hausdorff distances calculated for the roof UAS point clouds obtained by different software: Zephyr (a),

PhotoModeler Scanner (b), Drones2Map (top view and bottom up view) (c) and Visual SfM (d), using as reference data the mesh created based on GNSS measurements

The comparisons were made using the "Distance-Cloud/Mesh Dist" function from the "Tools" menu implemented into CloudCompare software, being calculated the Hausdorff distances between each point and the corresponding triangle surface. The calculated distances were summarized in Table 8.

\begin{tabular}{|c|c|c|c|c|c|}
\hline \multirow[b]{2}{*}{ Soft } & \multicolumn{2}{|c|}{ All points } & \multicolumn{2}{|c|}{ Most of the points } & \multirow[b]{2}{*}{$\begin{array}{l}\text { RMS } \\
{[\mathrm{cm}]}\end{array}$} \\
\hline & $\begin{array}{c}\text { Maximum } \\
\text { negative } \\
{[\mathrm{cm}]}\end{array}$ & $\begin{array}{c}\text { Maximum } \\
\text { positive } \\
{[\mathrm{cm}]}\end{array}$ & $\begin{array}{c}\text { Maximum } \\
\text { negative } \\
{[\mathrm{cm}]}\end{array}$ & $\begin{array}{c}\text { Maximum } \\
\text { positive } \\
{[\mathrm{cm}]}\end{array}$ & \\
\hline Zephyr & -28.1 & 24.2 & -9.5 & 7.7 & 5.3 \\
\hline Visual SfM & -84.6 & 89.5 & -16.8 & 24.9 & 16.1 \\
\hline $\begin{array}{c}\text { PhotoModeler } \\
\text { Scanner }\end{array}$ & -19.4 & 22.5 & -6.8 & 9.1 & 7.7 \\
\hline Drones2Map & -32.1 & 26.3 & -3.3 & 5.5 & 5.7 \\
\hline
\end{tabular}

Table 8 . The residuals of the roof UAS point clouds obtained by using different software

\subsection{Comparing the building facades created by different software}

To determine the accuracy of the 3D point clouds derived by using different software for the building facades, we will use the comparison method between the point cloud and a reference mesh surface, considered for this case study the mesh created based on the TLS data.

The comparisons were also made in CloudCompare software, the calculated distances being summarized in Figure 11.

Also the CAD 3D model created for the front facade of the building using the PhotoModeler software was compared with the TLS mesh, after a subsampling process was applied for each patch of the mesh using "Mesh-Sample Points" function, a standard deviation of $3.4 \mathrm{~cm}$ being obtained (Figure 12).

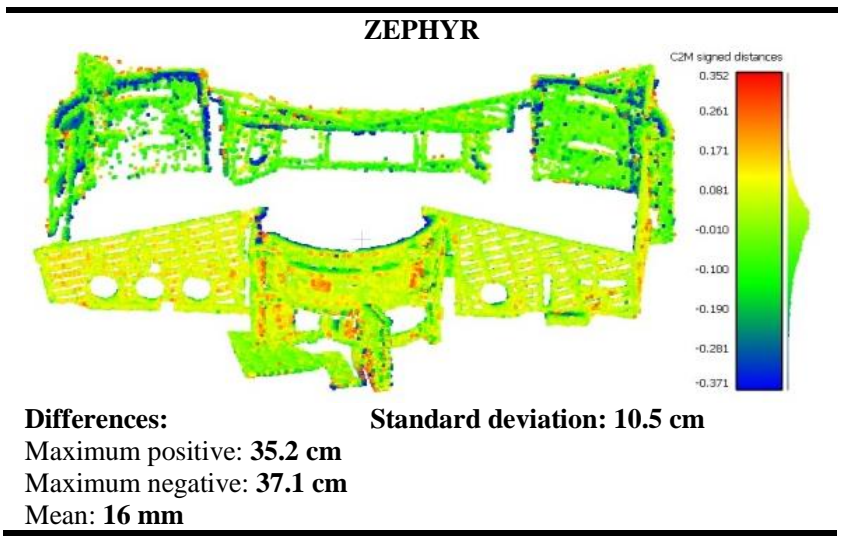

PHOTOMODELER SCANNER

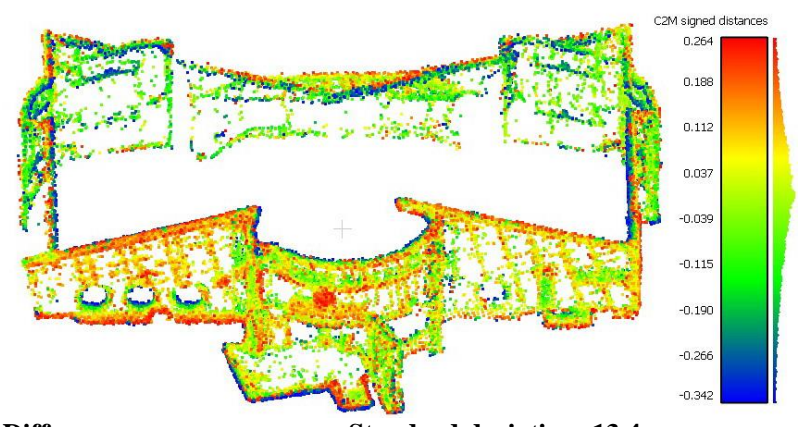

Differences:

Standard deviation: $13.4 \mathrm{~cm}$

Maximum positive: $26.4 \mathrm{~cm}$

Maximum negative: $34.2 \mathrm{~cm}$

Mean: $\mathbf{1 4} \mathbf{~ m m}$

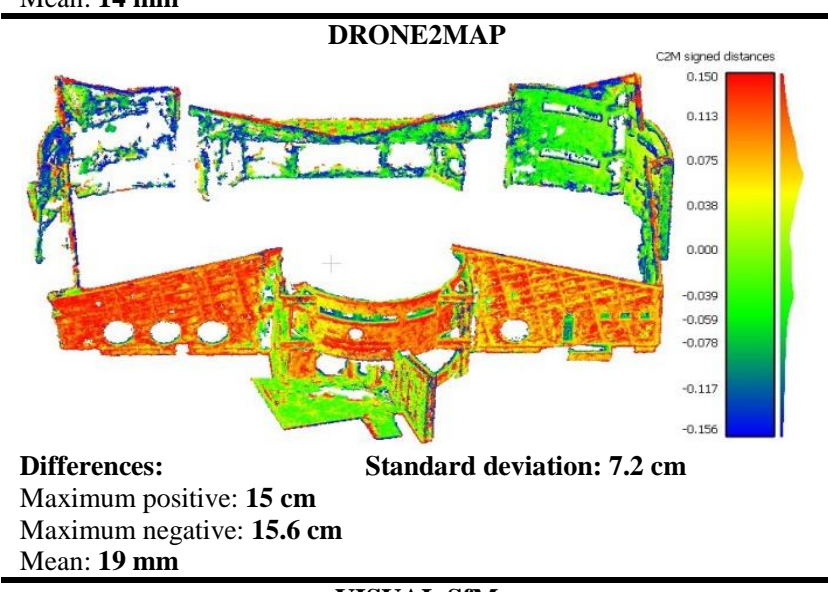

Mean: 19 mm
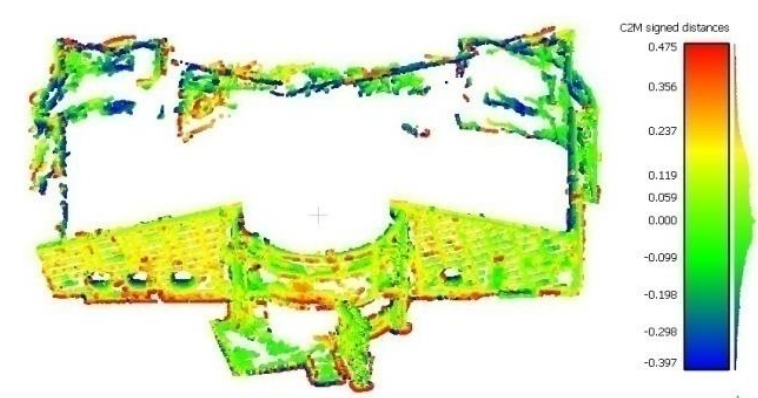

Differences:

Standard deviation: $17.0 \mathrm{~cm}$

Maximum positive: $47.5 \mathrm{~cm}$

Maximum negative: $39.7 \mathrm{~cm}$

Mean: 19 mm

Figure 11. The residuals of the facades UAS point clouds obtained by using different software 


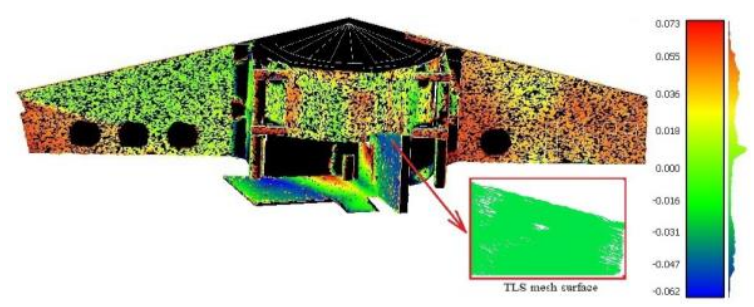

Figure 12. The Hausdorff distances calculated for the sample points generated for the front facade

\section{CONCLUSIONS}

This article presented a metric evaluation of automatically generated point cloud based on UAS images acquired with a low-cost platform, i.e DJI Phantom 3 Standard, using different software with the capability of automatically orienting the images.

All the tests were conducted under these assumptions:

- the interior and exterior parameters of the camera are unknown and have to be calculated by a self-calibration process (except the PhotoModeler Scanner software);

- only one image data set have been acquired with inclined camera axis towards the building facades (approximately $\left.45^{0}\right)$

- only 3 control points have been used for the indirect georeferencing process at the end of the bundle adjustment;

The roof's structure is a complex geometric form of a hyperbolic paraboloid and was approximated using least square method using different software. Obviously, the GNSS derived parameters of the hyperbolic paraboloid are best fitting. There are sensible differences between the results of using different software compared with the GNSS reference. Maximum corrections for the normal altitudes with respect to GNSS are in range of $[15 \div 80 \mathrm{~cm}]$ proving that the automatically generated point clouds are very noisy.

A quick analysis of the adjustment results can be made by visually inspecting the retrieved camera exterior orientation parameters (EO) (Figure 13). The visual analysis of the EO reveals some problems for the SfM tools.

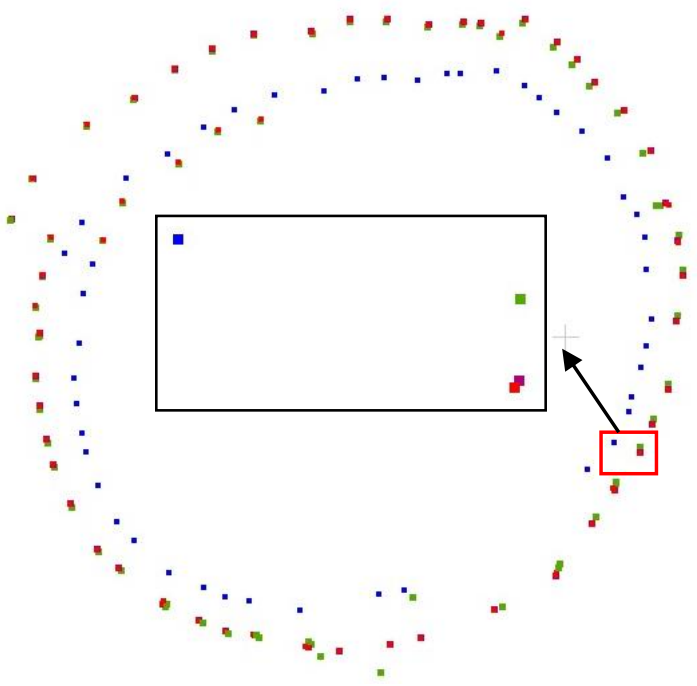

Figure 13. Visual analysis of the camera EO computed by using different software: PhotoModeler Scanner using only EXIF information (blue), PhotoModeler Scanner using calibrated camera (purple), Zephyr (red) and Visual SfM (green)
Regarding the interior orientation parameters, there is only one radial parameter $(\mathrm{k} 1)$ in Visual SfM, two radial parameters $(\mathrm{k} 1$ and $\mathrm{k} 2)$ in Zephyr and three radial parameters (k1, k2 and k3) in Drone2Map solved by a self-calibration bundle adjustment.

Checking the performances in terms of computed object coordinates based on image measurements, despite the point clouds oscillations with respect to a best fitting surface or a reference surface, the results are often positively surprising.

Taking into account the process automation, the processing time, the density of the point cloud and not the last the accuracy, we can order the study software starting with the best one as: Drone2Map, Zephyr, PhotoModeler Scanner and Visual SfM.

Further tests have to be carried out, considering the camera parameters calculated by a test field calibration for example and using the information of control points as constraints in a rigorous bundle block adjustment in order to obtain reliable results for images orientations. While you can never replace a traditional survey, drones can augment it in a way that provides significantly more value for the nearly same effort and cost.

\section{REFERENCES}

Barazzetti L., Scaioni M., Remondino F., 2010. Orientation and 3D modelling from markerless terrestrial images: combining accuracy with automation. The Photogrammetric Record 25(132), pp. 356-381.

Bolognesi, M., Furini, A., Russo, V., Pellegrinelli, A., Russo, P., 2015. Testing the low-cost potential in 3D cultural heritage reconstruction. The International Archives of the Photogrammetry, Remote Sensing and Spatial Information Sciences, Volume XL-5/W4, pp. 229-235.

Ghilani, C. D., Wolf, P. R. 2006. Adjustment computations. Spatial Data analysis, Fourth Edition, John Wiley\&Sons Inc., U.S.A, ISBN 978-0-471-69728-2.

Kersten, T., Mechelke, K., Maziull, L., 2015. 3D model of al zubarah fortress in Qatar-Terrestrial laser scanning vs. dense image matching. International Archives of the Photogrammetry, Remote Sensing Spatial Information Science, Volume XL5/W4, pp. 1-8.

Oniga, V.E., Chirila, C., Şutu M., 2012. Terrestrial laser scanner surveying versus total station surveying for $3 \mathrm{D}$ building model generation. Scientific Journal Mathematical Modelling in Civil Engineering, No. 8, pp. 168-177.

Oniga, V. E., Chirila, C., Macovei, M. 2016. Low-cost unmanned aerial systems in cadastral applications, $16^{\text {th }}$ International Multidisciplinary Scientific Geo Conference, SGEM 2016, Albena, Bulgaria, Book 2 Informatics, Geoinformatics and Remote Sensing, Volume II, , ISSN: 13142704, DOI: 10.5593/sgem2016B22, pp. 947-954.

Remondino, F., Del Pizzo, S., Kersten, T., Troisi S., 2012. Lowcost and open-source solutions for automated image orientation - a critical overview. Lecture Notes in Computer Science, 7616: $40-54$.

Nex F, Remondino F., 2013. UAV for 3D mapping applications: a review. Applied Geomatics 6(1): pp. 1-15. 